\title{
The Relationship Between Age and Arachnoid Depressions in Humans
}

\section{Rajitha Sivakumaran}

\begin{abstract}
The human skeletal collection housed in the Department of Anthropology at the University of Alberta was used to determine the relationship between age and the occurrence of arachnoid depressions on the endocranial aspect of the skull. There were significant differences between the total number of arachnoid depressions found on the vaults of juveniles, adolescents, and adults. When mean ages were compared with total number of arachnoid depressions on the vault, a significant relationship did not emerge. When age was grouped into nine-year intervals to counteract the effect of idiosyncratic variation, the mean number of depressions increased with age, as did the maximum number of arachnoid depressions. The frequency of older individuals without arachnoid depressions was low. Older individuals were more likely to have larger and deeper arachnoid depressions. There were no sex-based differences in the expression of arachnoid depressions. There were no significant differences between archaeological, bistoric, and modern samples or between pathological and healthy individuals. Although this study verifies the association between arachnoid depressions and senescence, the presence of arachnoid depressions is highly variable and cannot be used reliably as an indicator of chronological age or even as a sign of senescence.
\end{abstract}

$\mathrm{T}$ he brain is encompassed by three membranes. Beneath the skull lies the dura mater, followed by the arachnoid mater in the middle and the pia mater closest to the brain (Gosling et al. 1985). Of interest to this study is the arachnoid membrane, a thin, avascular layer separated from the pia mater by the subarachnoid space.

A review of the earliest descriptions of the arachnoid membrane by Adeeb et al. (2013) is summarized here. The earliest descriptions were made by Herophilus, the father of anatomy, in the 3 rd century B.C., and although many studies have been conducted on it since, one feature has received little attention: arachnoid depressions. These structures, also known as Pacchionian bodies, occur on the endocranial aspect of the skull. These "imprints" have been mentioned since ancient times under the name "glandulae" but largely ignored since the advent of modern medicine (Adeeb et al. 2013, 19). They were described first by Vesalius in 1543 as structures occurring in the region of the bregma of the cranial vault. In 1704, Pacchioni documented the presence of large depressions on the vault in the region of the sagittal sinus. These structures occur predominantly at the intersecting point of the sagittal and coronal sutures in the region of the superior sagittal sinus, but they have been documented at the lateral lacunae of the sagittal sinus, along the transverse sinus, and in anterior and middle cranial fossae as well (see Figure 1; le Gros Clark 1920; Grzybowski et al. 2007; Haybaeck et al. 2008).

As the name implies, these structures are dented regions on the bone surface of circular, ovoid, or irregular form and varying in size and depth. Figure 2 shows a skull of an aged individual from the $U$ of $A$ collection exhibiting arachnoid depressions along the sagittal suture. The enlarged images show their variation in size and depth in a single individual.

The origin of arachnoid depressions is debated. The imprints begin as arachnoid villi; these are protrusions on the exterior of the arachnoid mater and are observable microscopically during the late fetal stage (Adeeb et al. 2013). At this point, they remain separate from dural venous sinuses and lacunae (e.g., they have not penetrated the walls of these structures yet). With time, they become complex structures and their location be- 

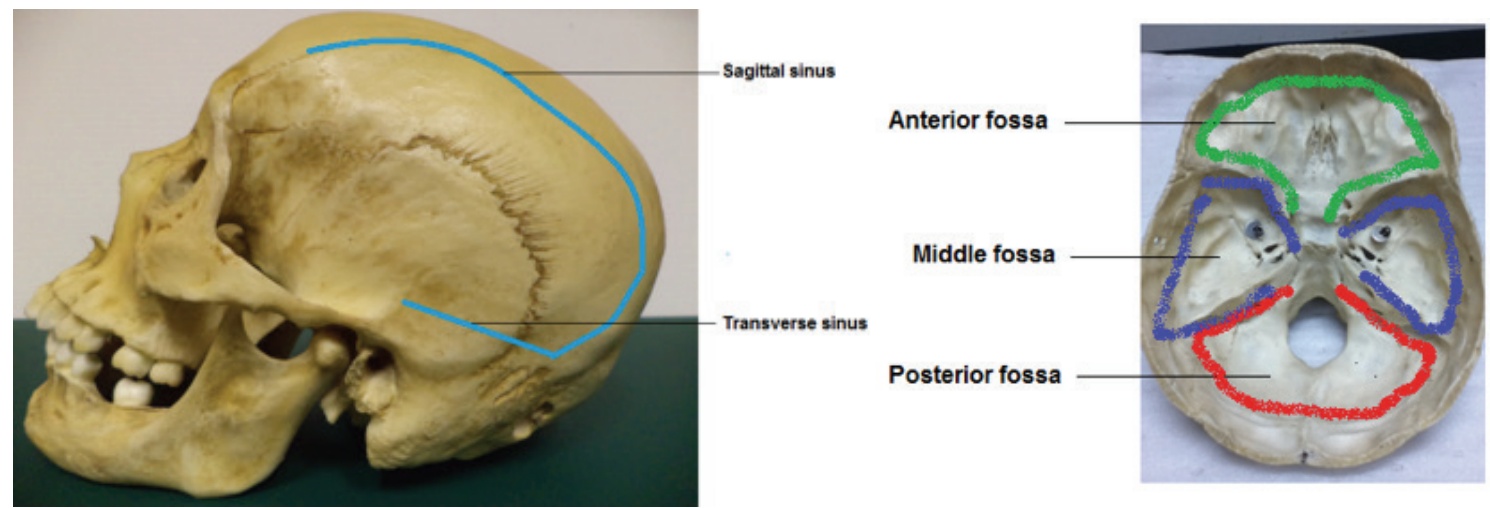

Figure 1: Superior sagittal sinus and transverse sinus of the vault (left) and anterior and middle cranial fossae of the cranial base (right).

comes (mostly) restricted to the region of the superior sagittal sinus. With the growth of the child (at ca. 18 months old), macroscopic observation becomes possible. The villi multiply in number, becoming structurally complex. At this point, they are referred to as arachnoid granulations. When these granulations reach a significant size, they leave imprints on the endocranial surface of the cranium, creating arachnoid depressions.

Children under the age of 10 do not possess these depressions (Basmajian 1952), and neither do many animals. This led Weed (1914) to the conclusion that Pacchionian bodies were skeletal indicators of pathology. This, however, is not the case. The presence of these imprints is related to the upright posture of older humans: "a negative pressure in the dural sinuses... a suction force... causes the arachnoid granulation to enlarge and bulge into the lumen" (Adeeb et al. 2013, 25). Early authors like Rokitansky in 1844 claimed that the depressions emerged as fibrous thickenings of the arachnoid, which consequently left imprints on the skull, while others like Cruveilhier, ca. 1847, believed that subarachnoid tissue gave rise to them. At the present time, the 1875 work of Key and Retzius is accepted. They described these structures as an extension of both the arachnoid membrane and subarachnoid tissue. Although the origin of arachnoid depressions is debated, its function is not. Pacchioni attributed a lymphatic function (e.g. draining of fluids) to these depressions. Two other authors, Fantoni in 1738
Figure 2: Arachnoid depressions of varying size and depth on the endocranial surface of the vault of a modern-day older adult from the University of Alberta skeletal collection.
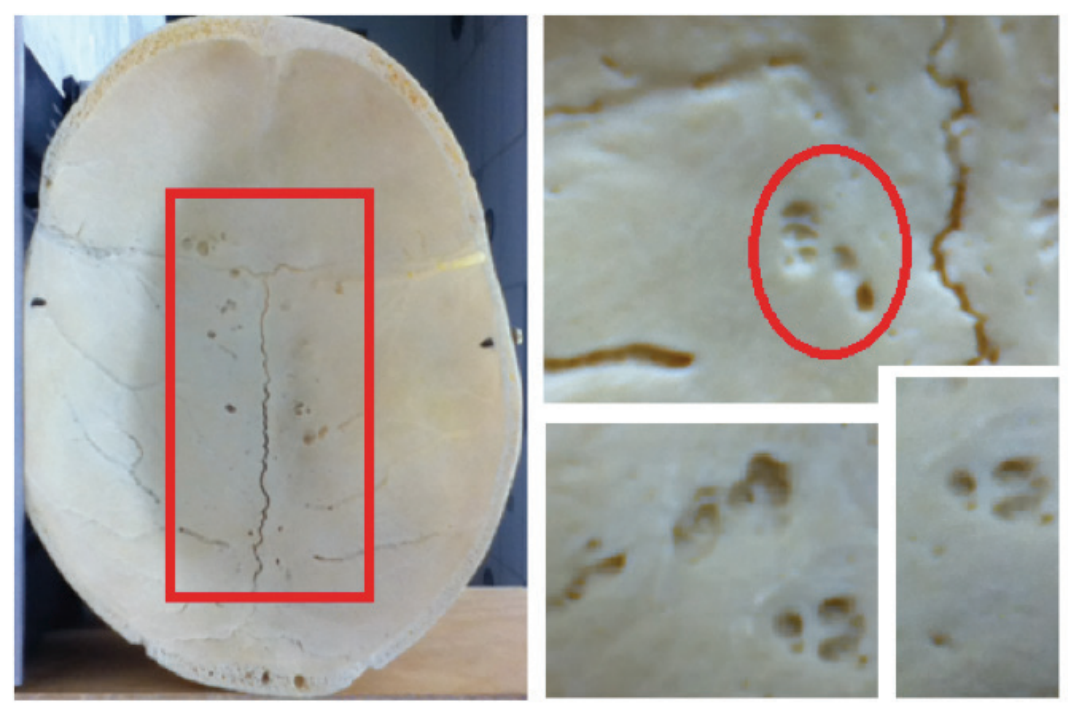
and Faivre in 1853, improved this early hypothesis by describing the role of arachnoid depressions in the absorption of intracranial humor or fluid. Key and Retzius defined this fluid as the cerebrospinal fluid (CSF; Adeeb et al. 2013).

These bodies are of osteological significance as they appear on bone and can be observed on skeletal remains. There are vague hints in the literature (e.g., Basmajian 1952) regarding the possibility that these structures increase in number, size, and depth with age. Thus, a senescent individual $(60+$ years $)$ will tend to exhibit more arachnoid depressions than a young adult. However, authors like von Luschka in 1855 (cited in Adeeb et al. 2013) report Pacchionian bodies are present in everybody, which if true, diminishes the prospect of using these structures as age indicators (Adeeb et al. 2013). On the other hand, Roche and Warner (1996) report that arachnoid depressions occur in one in 100 people.

Basmajian (1952) carried out the first and only study on this topic. His research revealed no statistically significant relationship between arachnoid depressions and age. People of the same age and sex had very different degrees of marking (scoring method used by the author) or expressions of arachnoid depressions. Some men of advanced age had a large degree of marking while other older adults had smaller scores. Despite this, a general increase in size was noted with age. The average degree of marking was much greater in age groups representing individuals over the age of 70 years compared to those who were 30 years and under. Within the former group, some individuals had numerous deep depressions while the opposite was observed in others. Basmajian (1952) concluded that arachnoid depressions could not be used as an indicator of age in a medicolegal setting.

Since only one modern study has been done on the topic, this project was undertaken to reevaluate the results of Basmajian (1952) and test for differences in the expression of arachnoid depressions across different ancestral groups. The potential application of this feature as an estimator of age was explored.

\section{Methods}

\section{Research questions}

The objective of this study was to conduct a thorough analysis of arachnoid depressions in humans and answer the following questions:

- Is there a positive correlation between the presence of arachnoid depression and age? Specifically, are they significantly associated with senescence or will young individuals exhibit them as well?

- Is there a correlation between arachnoid depression size, depth, and age?

- Where on the endocranial surface (vault and cranial base) do arachnoid depressions predominantly occur? Does it occur more often on the vault or the cranial base? Are there specific areas that exhibit these structures more frequently?

- Are there sex-based and/or populationbased differences in the expression (e.g. presence or absence) of arachnoid depressions?

- Does the time period (e.g. archaeological, historical, and modern) to which an individual belongs influence expression of arachnoid depressions?

- How does pathology impact expression of arachnoid depressions?

\section{Sample}

The skeletal collection housed in the Department of Anthropology at the University of Alberta was used to conduct this analysis ( $\mathrm{n}=$ 65). Adult males and females made up 53.8\% $(\mathrm{n}=35)$ and $38.5 \%(\mathrm{n}=25)$ of the sample, respectively. Two adolescents, four juveniles and one neonate were also included in the sample. The collection is predominantly comprised of homeless individuals from the Indian subcontinent (Calcutta), but several subjects of European, African, indigenous North American, and aboriginal Australian ancestry were included as well. 
Table 1. Scoring system for documenting size and depth of arachnoid depressions.

\begin{tabular}{ccc}
\hline Score & Size & Depth \\
\hline 1 & Small; $<1.5 \mathrm{~mm}$ & Shallow, no diploë exposure \\
2 & Moderate; $1.6-3.0 \mathrm{~mm}$ & Shallow, with diploe exposure \\
3 & Large; $>3.0 \mathrm{~mm}$ & Moderately deep; $0.5-1.0 \mathrm{~mm}$ \\
4 & - & Deep; $>1.0 \mathrm{~mm}$ \\
\hline
\end{tabular}

\section{Data collection}

A database was created using Microsoft Excel and SPSS, and the following information was collected for every specimen when possible: presence or absence of arachnoid depressions; age, following Meindl and Lovejoy's (1985) method for ectocranial sutures for adults and dental aging for subadults (Ubelaker 1978, cited in Scheuer and Black 2004), with the midpoint of the ranges used in statistical analyses; sex, following Acsadi and Nemeskeri's (1970) scoring system, supplemented by forehead slope, prominence of brow ridges and muscle markings, chin shape, gonial eversion, gonial angle, ramus length, extension of the zygomatic arch as the supramastoid crest and presence of frontal and parietal bossing (sex was not determined for the five subadults); ancestry, signs of pathology, vault weight and broad categories for time period (e.g. archaeological, historical, and modern); and number, location, shape, size, and depth of arachnoid depressions on the vault and cranial base for the 29 cases where it was possible to view the endocranial aspect.

\section{Scoring system}

A scoring system was used to document size and depth (in $\mathrm{mm}$ ) since obtaining actual measurements is difficult considering the small size of the depressions (see Table 1). The scores are based on arbitrary cut-off points, decided by the author after examining the amount of variation in size and depth across individuals.

\section{Zones}

Arachnoid depressions have been known to occur predominantly in the lateral lacunae along the superior sagittal sinus (le Gros Clark 1920). Haybaeck et al. (2008) noted that large granula- tions (approx. $2.5 \mathrm{~cm}$ ) occurred in the transverse sinuses of (mostly) individuals over the age of 65 years. They have been documented in the anterior and middle cranial fossae and posterior temporal bone as well (Chen et al. 2010). In order to map approximately where arachnoid depressions occur and verify previous research, the vault and cranial base of the skull were divided into numbered regions as shown in Figure 3. Every arachnoid depression was assigned a zone.

\section{Conflicting age estimations}

As mentioned, adult age estimations were determined using Meindl and Lovejoy's (1985) method using vault and lateral-anterior sutures A sample of 43 subjects for whom both vault and lateral-anterior average ages were available were used to show the conflicting results produced by these methods. The minimum and maximum ages for vault sutures were 30.5 and 75.0 years, respectively, and the mean age was $38.3 \pm 7.7$ years. For the lateral-anterior sutures, the minimum and maximum ages were 41.1 and 75.0 years, respectively, and the mean age was $52.8 \pm 6.1$ years. According to these mean ages, the lateral-anterior estimates indicate a significantly older sample than the vault ages, as shown by an independent T test $(\mathrm{t}=-10.415, \mathrm{df}=84, p=.000)$. Meindl and Lovejoy (1985) report that age estimations based on lateral-anterior sutures are more reliable, and thus, the lateral-anterior midpoint ages were used in most of the statistical analyses. These midpoint ages were arbitrarily grouped into nine-year periods to minimize the effects of idiosyncratic variation and reveal any general trends present (Table 2). Assigning an individual to a group depended on their lateral-anterior midpoint age, as well as other skeletal indicators (e.g., unfused basilar suture or age-related alveolar resorption). 
Figure 3: Zones used for mapping the location of arachnoid depressions on the vault and cranial base.
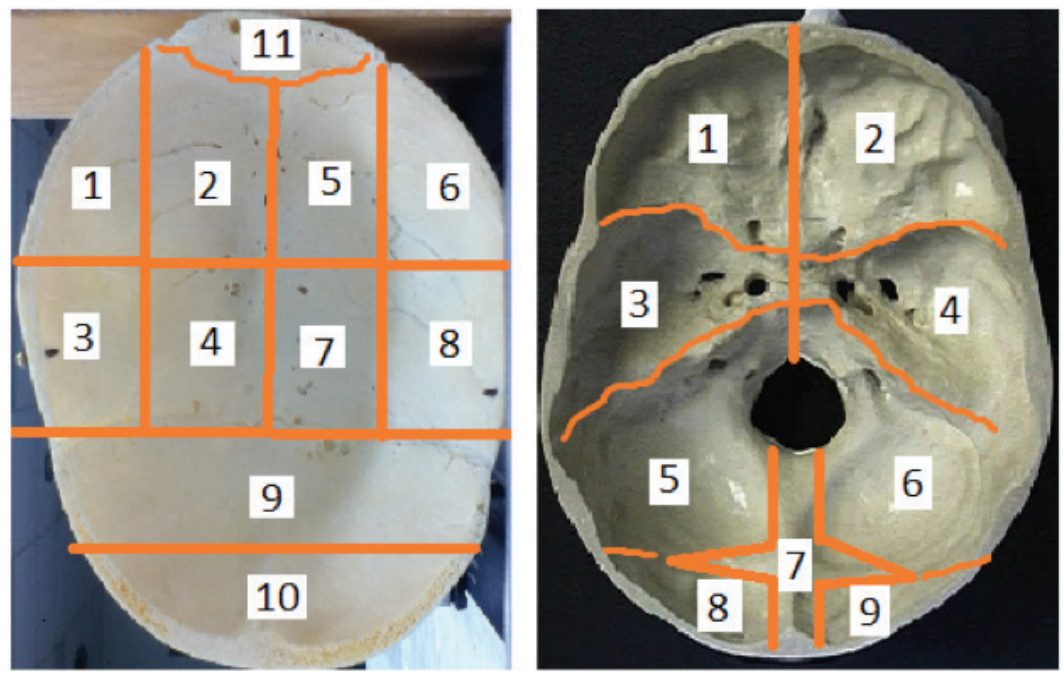

\section{Statistical analyses}

Non-parametric tests were the main type of statistics used in this study due to the small sample size (e.g. Chi-square, Mann-Whitney and Kruskal-Wallis tests). Regression analysis, coefficient correlations $\left(r\right.$ and $r^{2}$ ) and standard error of estimate were reported, as well. Most of these tests used the number of arachnoid depressions found on the vault, rather than the cranial base, to test for increase in frequency with age.

\section{RESULTS}

\section{Arachnoid depression and age}

Out of the 65 subjects included in this study, 52 , or $80 \%$, had arachnoid depressions on the vault, cranial base, or both. The neonate, all

Table 2. Adult and subadult age groups based on cranial sutural age and skeletal indicators.

\begin{tabular}{cc}
\hline Age group & Description \\
\hline 1 & $\leq 10$ years \\
2 & $11-20$ years \\
3 & $21-30$ years \\
4 & $31-40$ years \\
5 & $41-50$ years \\
6 & $51-60$ years \\
7 & $>60$ years \\
\hline
\end{tabular}

four juveniles, and one of the two adolescents did not exhibit arachnoid depressions. The other adolescent, whose age was estimated to be 18 years, had five depressions on the vault, which is low considering that the maximum number was 55 depressions in an individual aged 50-60 years. A Kruskal-Wallis test performed on 40 individuals showed that there were significant differences between the total number of arachnoid depressions found on the vaults of juveniles, adolescents, and adults $\left(\chi^{2}=8.017, \mathrm{df}=2, p=.018\right)$. The test results support the conclusions of previous studies (e.g., Basmajian 1952); arachnoid depressions develop with increasing age. In the present case, the results were based on a rather small subadult sample size, making the reliability of the conclusions somewhat questionable. As lateral-anterior age estimations indicate that the population is composed mostly of senescent individuals, the results verify the idea that arachnoid depressions are associated with older adults.

When mean ages were compared with total number of arachnoid depressions on the vault, a significant relationship did not emerge $(\mathrm{r}[19]=$ $.025, p=.457, \mathrm{~N}=21)$. A significant linear relationship does not exist between mean age determined from vault sutures and the total number of arachnoid depressions found on the vault $(\mathrm{r}[25]=$ -.053, $p=.397, \mathrm{~N}=27$ ). Although a statistically significant relationship between the presence of 
Table 3. Descriptive statistics of age groups based on dental data (subadults) and lateral-anterior sutures (adults), and the number of arachnoid depressions on the vault. The mean number of arachnoid depressions, standard error and deviation, and minimum and maximum number of occurrences, and sample size

(N) are shown.

\begin{tabular}{ccccccc}
\hline Age group & Mean & Std. error & $\begin{array}{c}\text { Std. } \\
\text { deviation }\end{array}$ & $\begin{array}{c}\text { Minimum } \\
\text { no. }\end{array}$ & Maximum no. & N \\
\hline 2 & 2.50 & 2.50 & 3.54 & 0 & 5 & 2 \\
5 & 9.17 & 4.61 & 11.29 & 0 & 30 & 6 \\
6 & 12.95 & 3.23 & 14.10 & 0 & 42 & 19 \\
7 & 21.00 & 17.16 & 29.71 & 0 & 55 & 3 \\
\hline
\end{tabular}

Age groups: 1, birth to 10 years; $2,11-20$ years; 3 , 21-30 years; $4,31-40$ years; 5 , $41-50$ years; $6,51-60$ years; and 7 , greater than 61 years. Arachnoid depressions were not observed in group 1 and no data were available for groups 3 and 4 .

arachnoid depressions and age did not emerge, Table 3 reveals interesting patterns worth discussing despite being derived from a small sample size.

Here, age was grouped into nine-year intervals to counteract the effect of idiosyncratic variation in the expression of arachnoid depressions (Figures 4 and 5). The mean number of depressions increased with age, as did the maximum number of arachnoid depressions. This pattern is somewhat compromised by the fact that some individuals in every age group exhibit no depressions. So, for reasons unknown, it is possible for older individuals to not have arachnoid depressions. This may be explained by genetic or envi- ronmental factors, or both. When the sample was restricted to include only those of Indian descent in order to control for population-based variation, only three adults exhibited absence. Overall, the analysis demonstrated that most older individuals had arachnoid depressions.

Determining whether the presence, size, and depth of arachnoid depressions are a function of increasing age is dependent on accurate aging methods if age is unknown, as is the case in this study. Since there is large variation in cranial sutural union commencement, progression, and termination (e.g. Key et al. 1994), it is likely that a high degree of error is present in the age esti-
Figure 4: Number of arachnoid depressions present on the vault plotted against age groups derived from lateral-anterior sutures (subadult age groups were based dental aging), where 1 is birth to 10 years; 2, 11-20 years; $3,21-30$ years; 4 , 31-40 years; $5,41-50$ years; 6, 51-60 years; and 7, greater than 61 years. No data were available for group 4.

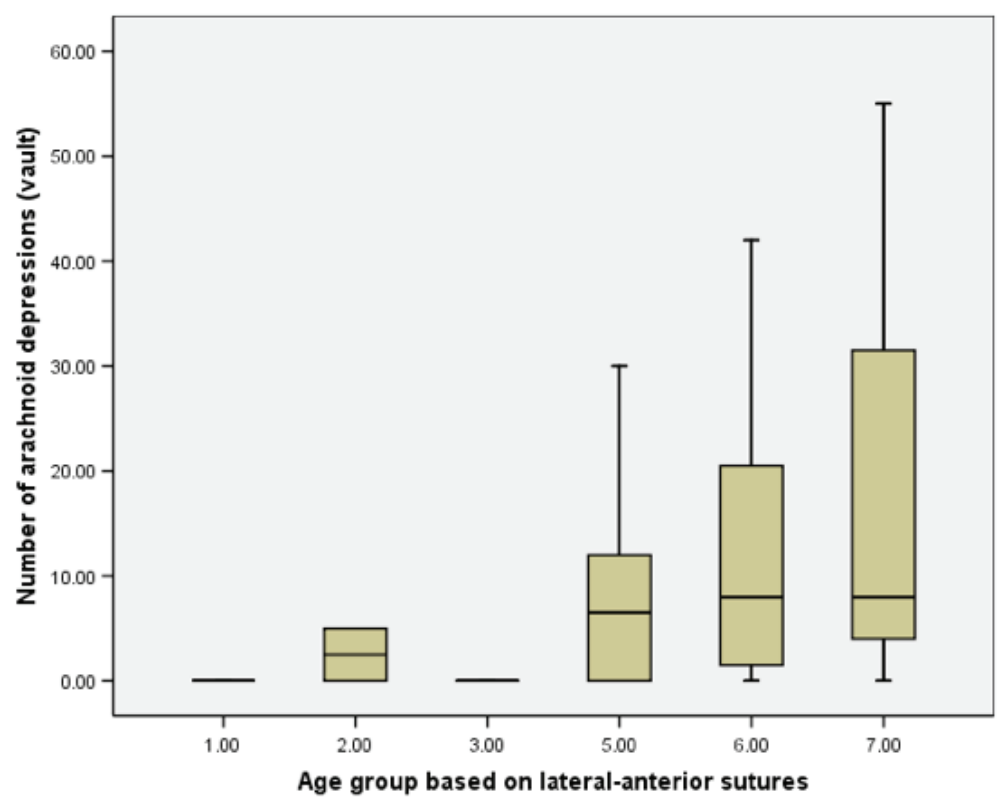


Figure 5: Number of arachnoid depressions present on the vault plotted against age groups derived from vault sutures (subadult age groups were based dental aging), where 1 is birth to 10 years; 2 , 11-20 years; 3, 21-30 years; 4, 31-40 years; $5,41-50$ years; 6,51 60 years; and 7 , greater than 61 years. Outliers are shown outside the box plot.

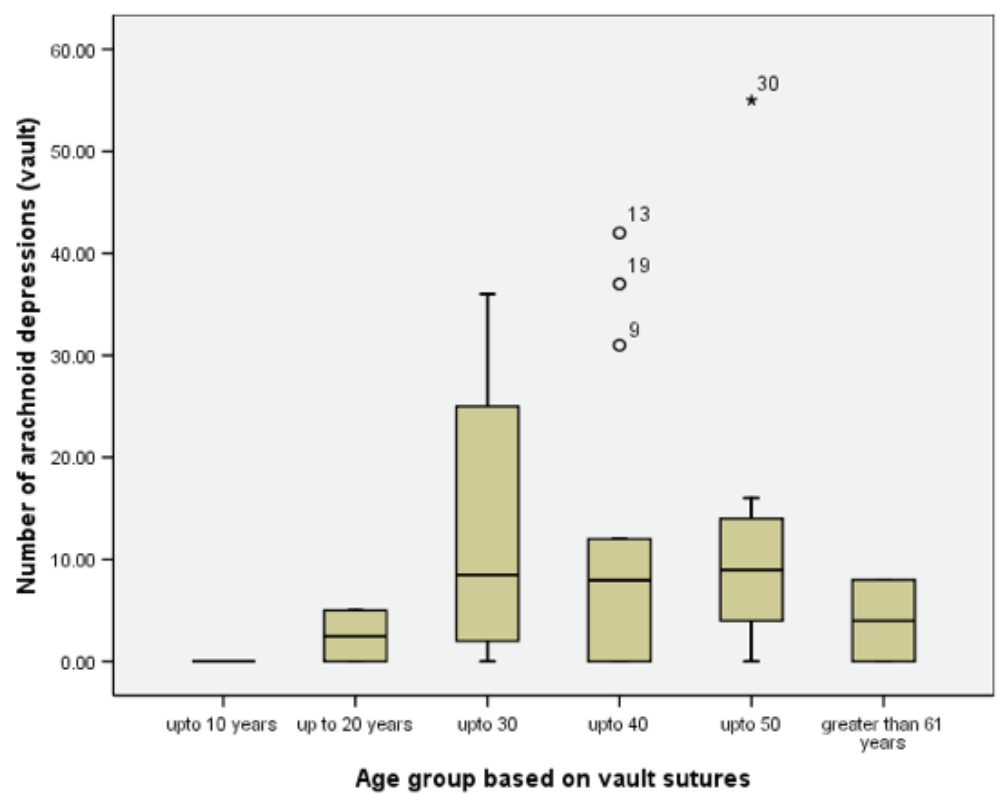

mations produced here. As this is only a preliminary study, dental analyses were not conducted to verify the ages. However, another potential estimate of age was included: weight of the vault. This is not a concrete method of aging; it relies on the idea that bone density decreases with age. If arachnoid depressions do correlate with age, a plot of vault weight against the number of depressions found on the vault may reveal patterns initially obscured by erroneous age estimations.

Figure 6 shows such a plot that illustrates two main clusters outlined in red and blue. The red cluster consists of individuals with a large number of arachnoid depressions, but relatively small weights, while the blue cluster shows varying weights, all of which are associated with less than 20 depressions. With the exception of the outlier, it appears that the greatest number of depressions occur in vaults weighing less than 250.0 grams. An unexpected positive correlation was seen between vault weight and number of depressions on the vault $\left(\mathrm{r}^{2}=.224\right)$, but this is only significant at the $0.05<p<0.10$ level ( $\mathrm{F}=$ 3.175, df $=2,22, p=.061)$. Removing the outlier produced the expected negative trend, but the correlation was no longer significant.

There are a number of possible explanations for the weak relationship between vault
Figure 6: Number of arachnoid depressions plotted as a function of weight in grams, showing two clusters, outlined in red and blue, and one outlier.

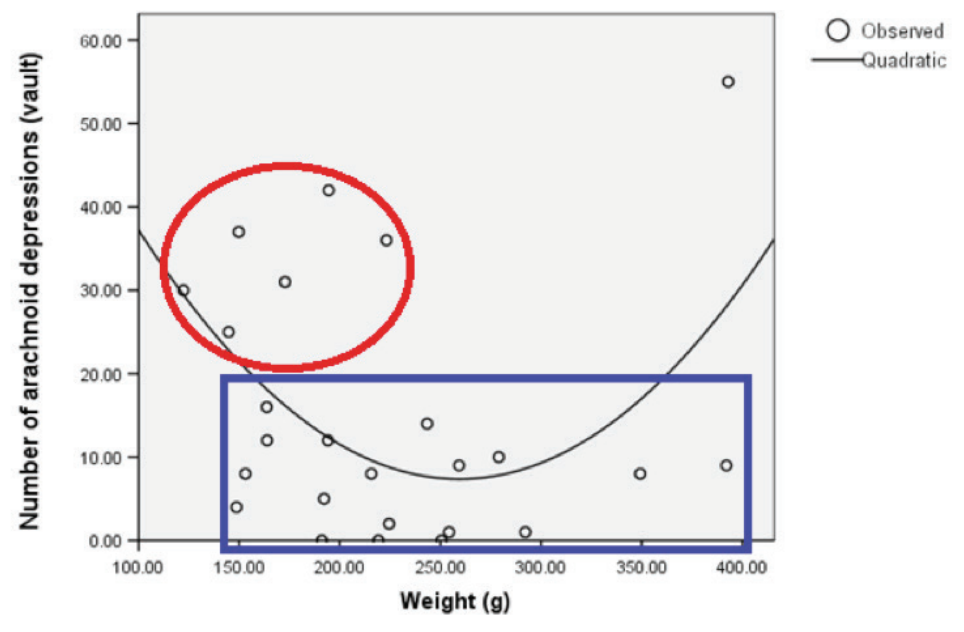


weight as a proxy for age and the number of arachnoid depressions on the vault. First, as demonstrated by the initial analysis and by Basmajian (1952), there may not be a correlation between age and the presence, number, size, and depth of arachnoid depressions. Secondly, although bone density decreases with age, the resulting density and weight depend on the total density, weight, or both (peak bone density) before reduction occurred. This is influenced by a number of things such as genetics and nutritional intake. Thus, even if all the older adults in this sample exhibited bone density loss with age, they may have had different peak densities. To test this, the sample was restricted to include only modern Indian adults, limiting for similar genetic and environmental conditions (e.g., homelessness in Calcutta, India). ANOVA indicates a significant relationship $(\mathrm{F}=4.373$, df $=20, p=.050)$. Here, however, sample size was reduced to 21 individuals and included subjects who did not possess any depressions. The correlation coefficient was weak $\left(r=.433, \mathrm{r}^{2}=.187\right)$. Although correlation coefficients are not the best indicators of a correlation and are sensitive to factors like sample size, the relationship between bone density (weight) and the number of arachnoid depressions on the vault remains unclear.

Other influential factors include the way a skull was cut. If the dimensions of the vaults used in this study are different, this will impact the weight as well. However, this is a minor source of error since, based on visual observation, the skull vaults roughly resembled one another in dimension. Vault thickness could have contributed to greater or lesser weights even if dimensions were similar. Simply observing the vault through the foramen magnum with a light source showed how opaque the bone was, and there was definite variation in this sample. The major source of potential error was variance in skull size. In other words, larger skulls would have larger vaults, which would weigh more. As this was a preliminary study, skull dimensions (e.g., length, width, shape) were not corrected for.

Another interpretation of the results indicates that the number of depressions may be correlated with skull density itself, rather than age. This may suggest that thinner cranial vaults are more likely to exhibit depressions. Future study comparing skulls of similar dimensions only would yield a more definitive understanding of the relationship between bone density and the number of arachnoid depressions.

\section{Arachnoid depression size and age}

Statistical analysis of lateral-anterior age and arachnoid depression size score did not reveal a significant relationship $(\mathrm{r}[19]=-.009, p=$ $.485, \mathrm{~N}=21$ ). Table 4 shows descriptive statistics using age groups. Although mean score increased from group 5 to 6 , an overall linear trend was not seen. The small sample sizes of groups 5 and 7 may have biased results.

\section{Arachnoid depression depth and age}

There is no significant relationship between the depth of arachnoid depressions and age ( $\mathrm{r}[20]$ $=.265, p=.117, \mathrm{~N}=22$ ). Table 5 compares age group with depth score. Although the oldest group (7) shows the greatest mean depth, the youngest group did not show the smallest depth.

Table 4. Descriptive statistics of age groups based on lateral-anterior sutures (adults), and the size (expressed as a score) of arachnoid depressions on the vault. The mean size score, standard error and deviation, and minimum and maximum scores, and sample size $(\mathrm{N})$ are shown.

\begin{tabular}{ccccccc}
\hline Age group & Mean & Std. error & $\begin{array}{c}\text { Std. } \\
\text { deviation }\end{array}$ & Minimum & Maximum & N \\
\hline 5 & 1.85 & .05 & .10 & 1.78 & 2.00 & 4 \\
6 & 2.14 & .09 & .35 & 1.67 & 3.00 & 15 \\
7 & 1.94 & .44 & .62 & 1.50 & 2.38 & 2 \\
\hline
\end{tabular}

Age groups: 5, 41-50 years; 6, 51-60 years; and 7, greater than 61 years. Size score: 1, small, up to 1.5mm; 2, moderate,1.6$3.0 \mathrm{~mm}$; and 3 , large, greater than $3.0 \mathrm{~mm}$. 
Table 5. Descriptive statistics of age groups based on lateral-anterior sutures (adults), and the depth (expressed as a score) of arachnoid depressions on the vault. The mean size score, standard error and deviation, and minimum and maximum scores, and sample size $(\mathrm{N})$ are shown.

\begin{tabular}{ccccccc}
\hline Age group & Mean & Std. error & $\begin{array}{c}\text { Std. } \\
\text { deviation }\end{array}$ & Minimum & Maximum & N \\
\hline 5 & 2.93 & .10 & .21 & 2.63 & 3.11 & 4 \\
6 & 2.90 & .11 & .43 & 2.08 & 4.00 & 15 \\
7 & 3.15 & .40 & .57 & 2.75 & 3.56 & 2 \\
\hline
\end{tabular}

Age groups: 5, 41-50 years; 6, 51-60 years; and 7, greater than 61 years. Size score: 1, small, up to 1.5mm; 2, moderate,1.6$3.0 \mathrm{~mm}$; and 3 , large, greater than $3.0 \mathrm{~mm}$.

Similar to the size results, the small sample sizes of groups 5 and 7 are potential sources of error.

There is a vague consensus that arachnoid depressions increase in size and depth with age, but no study has demonstrated this yet. This study applied a mathematical model, combining the scores for size and depth to produce a single score for 21 individuals that reflected both variables. The formula for calculating volume was adapted here, $\mathrm{V}=\mathrm{a} \times \mathrm{b} \times \mathrm{c}$ (length, width and height), to provide a rough estimation of the three-dimensional space occupied by each arachnoid depression. The hypothesis was that more of the older individuals would have the maximum size score of 3 , a depth score of 4 , and a combined score closer to 36, while the youngest adults would have values closer to 1 (minimum size and depth score $=1)$. Generally, amongst the younger adults, this appears to be the case (Figure 7 ), but due to the very broad age estimations produced by cranial sutures, the combined scores for the group with mean age 56.2 years (49-65 years) were difficult to interpret due to a large amount of variation. When considering only the mean scores of groups that consisted of more than one individual, a positive linear pattern was seen (Table 6).

\section{Locations}

Arachnoid depressions occurred more frequently on the vault compared to the cranial base. Of the open skulls, 29 crania exhibited arachnoid depressions on the vault, while 14 cases exhibited the depressions on the cranial base. The largest
Figure 7: A comparison of mean age in years derived from lateralanterior sutures with combined scores based on size and depth of arachnoid depressions of the vault.

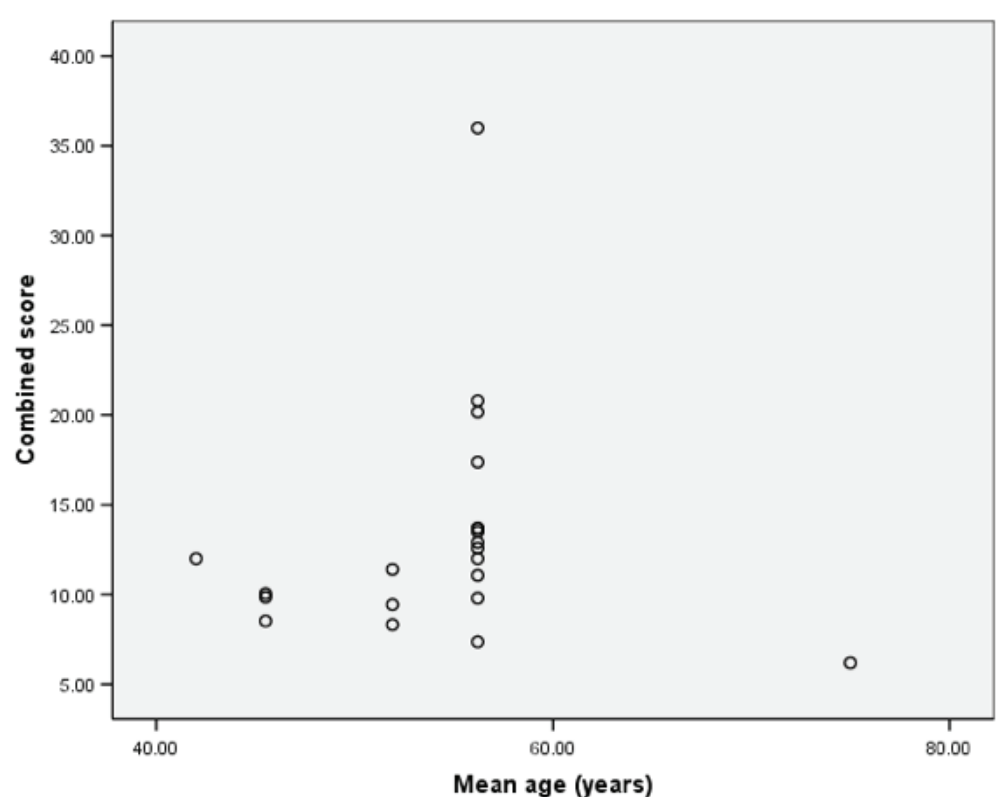

Mean age (years) 
Table 6. Descriptive statistics of ages based on lateral-anterior sutures and arachnoid depression scores for the vault. The mean score, standard error and deviation, and minimum and maximum scores, and sample size $(\mathrm{N})$ are shown.

\begin{tabular}{cccccccc}
\hline $\begin{array}{c}\text { Mean } \\
\text { age }\end{array}$ & Age range & $\begin{array}{c}\text { Mean } \\
\text { score }\end{array}$ & Std. error & $\begin{array}{c}\text { Std. } \\
\text { deviation }\end{array}$ & Minimum & Maximum & N \\
\hline 45.5 & $37-57$ & 9.47 & .48 & .83 & 8.52 & 10.05 & 3 \\
51.9 & $36-69$ & 9.72 & .90 & 1.56 & 8.32 & 11.40 & 3 \\
56.2 & $49-65$ & 15.45 & 2.01 & 7.25 & 7.36 & 36.00 & 13 \\
\hline
\end{tabular}

Age groups: 5, 41-50 years; $6,51-60$ years; and 7, greater than 61 years. Size score: 1 , small, up to 1.5mm; 2 , moderate, $1.6-3.0 \mathrm{~mm}$; and 3, large, greater than $3.0 \mathrm{~mm}$.

count reported for the latter was $14(\mathrm{n}=1)$ and more than half of the specimens $(57.1 \%)$ had less than five depressions. The largest count on the vault surface was $55(\mathrm{n}=1)$. Only $27.6 \%$ of samples had less than five depressions. Almost half of the sample $(41.4 \%)$ had more than 10 depressions. In only one case were depressions present exclusively on the cranial base, while 16 skulls had depressions on the vault but not on the cranial base. Thirteen skulls exhibited depressions on both the vault and cranial base. The total number of arachnoid depressions documented for the vault (a count of every observable depression on every skull) was 419 . The count for the cranial base, 76 , was much smaller.

In this study, the vault and the cranial base were considered separately, with regions in each being divided into zones to document approximately where arachnoid depressions occurred in the skull. The location of arachnoid depressions was represented by zones 1-11 and zones 1-9 for the vault and cranial base, respectively; the total count of arachnoid depressions occurring in all zones is shown in Table 7. Of the 419 depressions that occurred on the vault, zones 4 ( $\mathrm{n}=$ $96), 7(\mathrm{n}=105)$ and $9(\mathrm{n}=130)$ housed the most (79.0\%). For the cranial base, the largest amount of depressions was seen in zone $4(\mathrm{n}=28)$, followed by zone $5(\mathrm{n}=13)$. These zones accounted for approximately half of the depressions seen on the cranial base $(53.9 \%)$.

\section{Sex-based differences}

Due to unequal sample sizes, a MannWhitney test was used to assess if the total count of arachnoid depressions on the vault was sig-
Table 7. Zone of the vault and cranial base and the total number of arachnoid depressions that occurred in these regions.

\begin{tabular}{cc}
\hline Zone & $\begin{array}{c}\text { Count of arachnoid } \\
\text { depressions }\end{array}$ \\
\hline Vault & \\
\hline 1 & 0 \\
2 & 21 \\
3 & 1 \\
\hline 4 & 96 \\
\hline 5 & 5 \\
\hline 6 & 1 \\
\hline 7 & 105 \\
\hline 8 & 0 \\
\hline 9 & 130 \\
10 & 60 \\
\hline 11 & 0 \\
\hline Total & 419 \\
Cranial base & \\
\hline 1 & 0 \\
\hline 2 & 0 \\
\hline 3 & 5 \\
\hline 4 & 28 \\
\hline 5 & 13 \\
\hline 6 & 8 \\
\hline 7 & 10 \\
\hline 8 & 2 \\
\hline 9 & \\
\hline Total & \\
\hline Total count is based on 29 skulls with \\
depressions on the vault and 14 skulls \\
with depressions on the cranial base. \\
\hline & \\
\hline & \\
\hline
\end{tabular}


nificantly different between the sexes. Fourteen males and fifteen females $\left(\mathrm{n}_{\text {total }}=29\right)$ were used. The average number of arachnoid depressions occurring on the vault was higher for males (16.4 and 12.4 for males and females, respectively). Variation in the number of depressions was greater in males as well, demonstrated by the standard deviations (17.4 and 10.1 for males and females, respectively). However, these means were not significantly different $(\mathrm{U}=103.5, p=$ .948). An independent $T$ test for unequal variances $(F=4.934, p=.035)$ was performed to verify the non-parametric results. The relationship was insignificant here as well $(\mathrm{t}=.754$, df $=20.6, p=$ .459). A Mann-Whitney test carried out on males ( $n=14$ and 15 for size and depth, respectively) and females $(\mathrm{n}=15)$ showed that there were no significant differences between size $(\mathrm{U}=100.00$, $\mathrm{p}=.827)$ and depth $(\mathrm{U}=99.00, p=.574)$ of arachnoid depressions between the sexes.

\section{Population-based differences}

Although the study sample was comprised predominantly of those from the Indian subcontinent ( $\mathrm{n}=33$, or $50.8 \%$ of sample), individuals of European, African, indigenous North American, and aboriginal Australian ancestry were included, as well. Unfortunately, only one individual from each of these groups was available for study. Ancestry was unknown for 26 individuals. The number of arachnoid depressions was documented on only 25 Indians, one European, and one African individual since the other skulls were unopened. A statistical analysis could not be conducted on such a deficient sample size. A larger sample size where different ancestral groups are represented in adequate quantities is needed before any conclusions can be made about the impact of genetics and inter-population variation in the expression of arachnoid depressions.

\section{Time period}

A Kruskal-Wallis non-parametric test was used to test whether the total number of arachnoid depressions varied between modern ( $\mathrm{n}=$ 23), historical $(\mathrm{n}=5)$ and archaeological ( $\mathrm{n}=$ 1) populations $\left(\mathrm{n}_{\text {total }}=29\right)$. Results were insignificant $\left(\chi^{2}=4.830\right.$, df $\left.=2, p=.089\right)$. There is a strong potential for biased results in this case, particularly with the archaeological sample, since an adequate size was not available for study.

\section{Pathology}

A Mann-Whitney test was used to assess whether pathology can increase or decrease the number of arachnoid depression in a person. In a sample of 29 individuals, five were categorized as pathological. There were no significant differences in the mean number of arachnoid depressions seen on the vault of normal and pathological individuals $(\mathrm{U}=51, p=.602)$.

\section{Discussion}

The relationship between age and the presence of arachnoid depressions on the endocranial skull was reexamined in an attempt to determine whether a positive correlation exists. The initial hypothesis was that the number, size, and depth of arachnoid depressions would increase with age. The results confirm this hypothesis, but the relationship is not statistically significant. There are a number of reasons why this might be the case. Firstly, as already demonstrated by Basmajian (1952), arachnoid depressions may not be related to age. This research indicates that there is great variability in the expression of these structures between different people and ages. For instance, a very old individual like case 999.2.84 a and b (documented age of 75 years) possessed only eight depressions. Although she had, on average, moderately deep depressions, their mean size was small. Thus, idiosyncratic variation may obscure patterns. Secondly, the collection was of unknown age, and ages had to be estimated using the problematic method of cranial suture closure. In the words of Singer $(1953,56)$, “...the age of the individual at death cannot be estimated from the degree of closure of the various cranial sutures, whether taken individually or collectively or whether observed exocranially or endocranially."

Furthermore, the age ranges were very broad, which in turn produced midpoint ages that are most likely to deviate considerably from the actual age. Thus, erroneous age estimations could have biased results. Lastly, the sample size was small, and the effect of this was most ap- 
parent when actual ages were organized into age groups. Some of these groups consisted of only a couple of individuals. If they are atypical in any way, results will be biased. Due to the small size, non-parametric tests were used to determine significance. These tests, although powerful, are more likely to dismiss a correlation as insignificant than parametric tests. Future research with an adequate sample size can remedy this.

Previous research on arachnoid granulations, with the exception of Basmajian (1952), did not focus on the potential application of these structures as indicators of senescence. Instead, early researchers were more concerned with documenting appearance, origin, structural organization, etc. This study has verified some earlier conclusions while questioning others. Although Basmajian (1952) reported that children under 10 years of age did not exhibit arachnoid depressions, le Gros Clark (1920) observed these on the cadavers of young children aged 1.5 to 4 years old in the lateral lacunae along the superior sagittal sinus. This study supports Basmajian's (1952) conclusions, but only one neonate and four juveniles were available for analysis.

Mapping the locations of arachnoid depressions offered a good way of verifying the accounts of previous research, which indicates that these structures occur predominantly in the lateral lacunae along the superior sagittal sinus (zones $2,4,5$, and 7 of the vault), at the junction of the sagittal and coronal sutures (zones 4, 7, and 9 of the vault), transverse sinuses (zones 7, 8, and 9), anterior (zones 1 and 2) and middle (zones 3 and 4) cranial fossae, and posterior temporal bone, (zones 5 and 6) (le Gros Clark 1920; Haybaeck et al. 2008). In this analysis, the highest frequencies of arachnoid depressions on the vault were seen along the anterior half of the sagittal sinus and at the junction of the sagittal and coronal sutures. On the cranial base, the right portion of the middle cranial fossa was home to more depressions than other zones. This study found depressions in the left portion of the posterior cranial fossa as well. Previous studies did not find any depressions in this region, but in the sample examined here it contained the second largest number of structures in the cranial base. Although Okamo- to et al. (1997) noted the presence of arachnoid granulations (the soft tissues which cause erosion of the inner table, creating arachnoid depressions) on the posterior cranial fossa, this was a histological observation. Depressions in the area of the transverse sinus occurred in significant quantities as well.

One last point of interest concerns pathology. Winkelman and Fay's 1930 study (cited in Adeeb et al. 2013) found that cadavers presenting agenesis of arachnoid villi suffered from hydrocephalus, a condition described as a buildup of fluid in the brain (Gutierrez et al. 1975). In cadavers with no signs of hydrocephalus, arachnoid villi were present. Since the villi give rise to the Pacchionian bodies, hydrocephalus reduced the probability of developing arachnoid depressions. Following this example, the pathological specimens of the collection were compared to healthy individuals (although since the assemblage was made up of largely homeless individuals, "healthy" is a relative term). Pathologies as severe as hydrocephalus were not noted; instead idiopathic anomalies like asymmetrical vault bones (e.g., parietals) and cranial base (e.g., posterior cranial fossa), and extra sutures were classified as pathological. Case 369 a and b showed diploic exposure on parts of the frontal and parietal bones. A 75-year-old forensic case, 999.2.84 a and $\mathrm{b}$, exhibited bony protrusions in the anterior and middle cranial fossae. In fact, this case was a consistent outlier in most of the statistical analyses. Although comparing pathological and normal individuals yielded no significant differences, this problem should be reevaluated with a larger pathological sample size.

\section{Conclusion}

Although arachnoid depressions are typically associated with senescence and are suspected to increase in size and depth with age, the only study done to date (Basmajian 1952) disputes this, and this study mostly supports Basmajian's conclusions. There were significant differences between the total number of arachnoid depressions found on the vaults of juveniles, adolescents, and adults, but when estimated mean ages were used, a significant relationship did not 
emerge. However, general, statistically insignificant patterns did arise. When age was categorized into groups to offset the effect of idiosyncratic variation, the mean number of depressions as well as the maximum number of arachnoid depressions increased with age. The frequency of older individuals without arachnoid depressions was low. A negative correlation between vault weight (as a proxy for age, since bone density and weight decrease during senescence) and number of depressions on the vault is seen with the removal of one outlier. Arachnoid depression size and depth did not increase with age, but when a combined score was produced representing both of these variables, it was shown that older individuals were more likely to have larger and deeper arachnoid depressions.

Approximate mappings of the depressions showed that they were most likely to occur on the vault rather than the cranial base. Nearly $80 \%$ of depressions occurred in zones 4, 7, and 9 on the vault, as demonstrated by current literature. There were no differences in the expression of arachnoid depressions based on sex, pathology, and time period. Analyses on inter-population variation were inconclusive. Although this study supports the association between arachnoid depression and senescence, it confirms the conclusion of Basmajian (1952). The presence of arachnoid depressions is highly variable and cannot be used reliably as an indicator of chronological age.

\section{ACKNOWLEDGEMENTS}

Special thanks are owed to Dr. Nancy Lovell for providing the idea for this paper and for reminding me how exciting the world of $\mathrm{pa}$ leopathology is.

\section{References Cited}

AcsÁd, György, JÁnos Nemeskéri, AND KornéL BALÁs. 1970. History of Human Life Span and Mortality. Budapest: Akadémiai Kiadó.

Adeeb, Nimer, Aman Deep, Christopher J.

Griessenauer, Martin M. Mortazavi, Koichi

Watanabe, Marios Loukas, R. Shane Tubbs, And Aaron A. Cohen-Gadol. 2013. "The intracranial arachnoid mater." Child's Nervous System 29(1):17-33.
Basmajian, John V. 1952. "The depressions for the arachnoid granulations as a criterion of age." The Anatomical Record 112(4):843-846.

Chen, Feng, Xue-fei Deng, Bin Liu, Li-na Zou, De-bin Wang, and Hui Han. 2011. "Arachnoid granulations of middle cranial fossa: A population study between cadaveric dissection and in vivo computed tomography examination." Surgical and Radiologic Anatomy 33(3):215-221.

Gosling, John A., P.F. Harris, J.R. Humpherson, I. Whitmore, and P.L.T. Willan. 1985. Atlas of Human Anatomy with Integrated Text. Philadelphia: Lippincott Company.

Grzybowski, Deborah M., Edward E. Herderick, Kapil G. Kapoor, David W. Holman, And STEven E. Katz. 2007. "Human arachnoid granulations: A technique for quantifying area and distribution on the superior surface of the cerebral cortex." Cerebrospinal Fluid Research 4(6):1-7.

Gutierrez, Yezid, Reinhard L. Friede, and William J. Kaliney. 1975. "Agenesis of arachnoid granulations and its relationship to communicating hydrocephalus." Journal of Neurosurgery 43(5):553-558.

Haybaeck, Johannes, Rene Silye, and Dov SofFER. 2008. "Dural arachnoid granulations and "giant" arachnoid granulations." Surgical and Radiologic Anatomy 30(5):417-421.

Key, C.A., Aiello, L.C., ANd Molleson, T. 1994. "Cranial suture closure and its implications for age estimation." International Journal of Osteoarchaeology 4:193-207.

Le Gros Clark, W.E. 1920. “On the Pacchionian bodies." Journal of Anatomy 55:40-48.

Meindl, Richard S., and C. Owen Lovejoy. 1985. "Ectocranial suture closure: A revised method for the determination of skeletal age at death based on the lateral-anterior sutures." American Journal of Physical Anthropology 68(1):57-66.

Oкамото, Kouichirou, Jusuke Ito, Susumu Tokiguchi, Tetsuya Furusaina, and Mamiko NishiHARA. 1997. "Arachnoid granulations of the posterior fossa: CT and MR findings." Clinical Imaging 21(1):1-5. 
Roche, James, AND Denise WARner. 1996. "Arachnoid granulations in the transverse and sigmoid Sinuses: CT, MR, and MR angiographic appearance of a normal anatomic variation." American Journal of Neuroradiology 17(4):677-683.

Scheuer, Loiuse, and Sue Black. 2004. The Juvenile Skeleton. New York: Academic Press.

Singer, RonAlD. 1953. "Estimation of age from cranial suture closure. A report on its unreliability." Journal of Forensic Medicine 1(1):52-59.

U.S. National Library of Medicine and the National Institutes of Health. 2012. "Meninges of the brain." Electronic resource, http://www.nlm.nih.gov/medlineplus/ency/ imagepages/19080.htm (accessed February 16, 2014).

WEED, LEWIS H. 1914. "Studies on cerebro-spinal fluid. No. III: The pathways of escape from the subarachnoid spaces with particular reference to the arachnoid villi." Journal of Medical Research 31:51-91. 